\title{
Normal Human Intestinal B Lymphocytes Increased Activation Compared with Peripheral Blood
}

Marion G. Peters, Heather Secrist, Kirk R. Anders, Geoffrey S. Nash, Shelly R. Rich, and Richard P. MacDermott

Washington University School of Medicine, St. Louis, Missouri 63110

\begin{abstract}
The state of activation of normal human intestinal mononuclear cells obtained from transplant donors was studied. Compared with PBMC, freshly isclated intestinal mononuclear cells expressed significantly more cell surface activation antigens on both $B$ and $T$ lymphocytes. Intestinal mononuclear cells contained significant numbers of immunoglobulin secreting cells immediately after cell separation. This population included CD5-positive $B$ cells that secreted predominantly IgA. Cells from the large bowel consistently revealed higher numbers of IgA secreting cells than cells from the small bowel. Thus, intestinal B cells are markedly activated in vivo compared with PBMC and this increased activation correlates with increased spontaneous antibody secretion. $B$ cells from the large intestine are more highly activated and secrete more antibody than do cells from the small intestine. The intestinal lamina propria lymphoid compartment exhibits a heightened state of activation that may be important for its distinct role in mucosal defense.
\end{abstract}

\section{Introduction}

Previous studies have demonstrated that human intestinal mononuclear cells (INT MNC) ${ }^{1}$ have increased spontaneous antibody secretion after $14 \mathrm{~d}$ in vitro culture (1). This enhanced secretion may be due to $B$ cell activation per se, increased $\mathrm{T}$ cell help, decreased suppressor cell activity, and/or an increase in secretion of $B$ cell growth and differentiation factors. Determining the mechanism(s) for this increased spontaneous antibody secretion is important, both for a better understanding of normal intestinal $B$ cell activation and for providing a basis for the understanding of $B$ cell activation and antibody secretion in disease states. Studies to date of normal INT MNC function have been hampered by the fact that only small numbers of INT MNC can be obtained, usually at the time of surgery from patients with colonic disease. Therefore limited studies can be performed and important variables such as medications, nutrition status, and the disease process itself may influence the results. In this paper we describe the use of

Address reprint requests to Dr. Marion Peters, Box 8124, Washington University School of Medicine, 660 South Euclid, St. Louis, MO 63110.

Received for publication 7 March 1988 and in revised form 24 January 1989.

1. Abbreviations used in this paper: INT MNC, intestinal mononuclear cells.

J. Clin. Invest.

(C) The American Society for Clinical Investigation, Inc.

$0021-9738 / 89 / 06 / 1827 / 07 \quad \$ 2.00$

Volume 83, June 1989, 1827-1833 transplant donor intestinal specimens, which now allows the examination of normal INT MNC from both large and small bowel.

We investigated the activation state of normal INT B cells by three methods. First, we quantitated the expression of activation markers on the plasma membrane using MAb. Second, we quantitated immunoglobulin-secreting cells (ISC) immediately after cell separation (time zero) and after various periods of time in culture. Finally, we have correlated these data with total and isotype-specific Ig secretion in culture. We find by all three criteria that normal INT B cells are more activated than PBMC. B cells from large bowel are more activated by these criteria than the equivalent cell population from small bowel. The presence of a highly activated population of lymphocytes at this intestinal mucosal surface may be an important mechanism of host defense and of normal bowel immunity.

\section{Methods}

Isolation of cells. PBMC were isolated from heparinized blood of normal healthy volunteers using Ficoll-Hypaque centrifugation (2). Normal small and large bowel INT MNC were obtained from surgically removed specimens from individuals donating organs for transplantation, at the same time as other donor organs were harvested. Warm ischemia time was $<45 \mathrm{~min}$, which is shorter than that for surgically removed specimens. Protocols were approved by the Human Use Study Committee at Washington University School of Medicine. Mononuclear cells from human intestinal mucosa were isolated as described (3). Briefly, the mucosa was dissected free, cut into fine pieces and washed repeatedly in calcium- and magnesium-free HBSS containing antibiotics. The minced pieces were stirred in multiple changes of media containing $0.75 \mathrm{mM}$ EDTA. After epithelial cells were removed, the tissue was incubated overnight in collagenase medium and $20 \%$ heat-inactivated pooled human serum. After collagenase digestion, cells were centrifuged through Ficoll-Hypaque, Percoll, and fetal bovine serum gradients. INT MNC contained $70 \pm 5 \% \mathrm{~T}$ cells, $12 \pm 3 \%$ B cells, and $8 \pm 4 \%$ monocytes. They were $>95 \%$ viable by trypan blue dye exclusion. Intestinal MNC contained 8-10\% plasma cells by morphologic staining. There were no epithelial cells in INT MNC as assessed by morphology or staining with antibody to Factor VIII (Dako Corp., Santa Barbara, CA) and keratin (Hybritech, San Diego, CA).

In some experiments, $B$ and $T$ cells were isolated by twice rosetting with 2-aminoethylthiouronium-bromide-treated sheep red blood cells (4). B cell enriched populations contained $>50 \%$ surface $\mathrm{Ig}(\mathrm{sIg})$ positive cells, $<1 \% \mathrm{E}$ rosette positive cells, with the remaining cells containing plasma cells, $5-15 \%$ monocytes, and $5-10 \%$ null cells. T cell enriched subpopulations contained $>95 \% \mathrm{E}$ rosette positive cells and $<1 \%$ sIg positive cells. CD5-positive B cells were obtained by cell sorting B cell-enriched INT MNC using Leu 1 (Becton Dickinson \& Co., Oxnard, CA) on a FACS (model 400; Becton Dickinson \& Co.).

Surface staining. The following MAbs were used: 4F2, which recognizes a $120-\mathrm{kD}$ cell surface protein that appears on activated cells (5); $5 E 9$, which recognizes the transferrin receptor (6); anti-TAC which recognizes a 55-kD protein of the IL 2 receptor (a kind gift of Dr. T. Waldmann, National Institutes of Health, Bethesda, MD) (7); and BA5, which recognizes the high molecular weight B cell growth factor receptor (kind gift of Dr. J. L. Ambrus, National Institutes of Health) 
(8). For single staining, $10^{6}$ mononuclear cells were incubated with the MAb for $60 \mathrm{~min}$ at $4^{\circ} \mathrm{C}$ in the presence of $1 \mathrm{mg} / \mathrm{ml}$ human $\mathrm{IgG}$ to block Fc receptors. After washing, positive cells were counted by fluorescent microscopy or by fluorescence-activated dual microcytometry (FACS). For microscopic evaluation, cells were incubated with latex beads for $45 \mathrm{~min}$ at $37^{\circ} \mathrm{C}$ and then washed three times before incubation with the MAb. When fluorescent microscopy was used, positive cells were determined by the total number of fluorescent cells which had not ingested latex beads (nonphagocytic cells) minus the background (number of cells positive with second antibody alone). For dual staining, cells were incubated as before with the MAb and washed. Phycoerythrin-conjugated goat anti-mouse Ig (Biomeda, Foster City, CA) was then added. After $60 \mathrm{~min}$ at $4^{\circ} \mathrm{C}$, the cells were washed and directly fluoresceinated Leu-1 or Leu-4 (T cells), Leu-12 (B cells), or Leu-M3 (monocytes, Becton Dickinson \& Co.) was added for 60 min at $4^{\circ} \mathrm{C}$. After washing, the cells were placed in $1 \%$ paraformaldehyde until they were analyzed. The percentage of positive cells was assessed by fluorescence activated dual microcytometry. Two-dimensional graphs were obtained and the percentage of $B$ cells, $T$ cells, or monocytes that were positive for activation antigens was measured using a computerized program. Initial studies revealed that gating out monocytes also removed the large preactivated lymphoblastoid cells. Large cells contain monocytes that are positive for $4 F 2$ and $5 E 9(5,6)$. Therefore for FACS studies, only those mononuclear cell populations with $<5 \%$ monocytes were used. In some experiments, positive cells were estimated by both fluorescent microscopy and FACS and similar results were obtained. The percentage of $B$ and $T$ cells were $12 \pm 3$ and $70 \pm 5 \%$, respectively. In some experiments $10^{6} \mathrm{MNC}$ were cultured with PWM (1:200 vol/vol, Gibco Laboratories, Grand Island, NY), for 3 and $5 \mathrm{~d}$, washed, and stained for various surface antigens as above.

Immunoglobulin secretion. $2 \times 10^{5}$ cells were cultured in complete media ( $10 \%$ FCS, $50 \mu \mathrm{g} / \mathrm{ml}$ gentamicin) in $200 \mu$ for $14 \mathrm{~d}$. After $14 \mathrm{~d}$, supernatants were assayed for IgG, IgA, and IgM by ELISA (9). For kinetic experiments, multiple wells were set up as above and supernatants from triplicate wells were removed at days $0,2,4,6,8,11$, and 14 and assayed for Ig. Cell viability was $>90 \%$ until day $7,50-70 \%$ until day 11 , and after $14 \mathrm{~d}$ in culture was $45 \%$.

ISC were assayed by a spot ELISA technique (10). Briefly, 96-well flat-bottom plates were coated with anti-human IgG, IgA, or IgM (Cappel Laboratories, Malvern, PA) overnight at $4^{\circ} \mathrm{C}$. The plates were then washed with PBS-Tween and cells, at varying dilutions in $1 \%$ BSA-RPMI, were added for $18 \mathrm{~h}$ at $37^{\circ} \mathrm{C}$. After washing with PBSTween, alkaline phosphatase-conjugated anti-human IgG, IgA, or IgM (Sigma Chemical Co., St. Louis, MO) was added for $3 \mathrm{~h}$ at $37^{\circ} \mathrm{C}$. The plates were washed once again with PBS-Tween and the substrate, 5 bromo-4-chloro 3-indolylphosphate 2-amino-2-methyl-1-propanol in 3\% agarose was added for $6 \mathrm{~h}$. Because the final substrate is added in agarose, Ig secreted by the B cells at the start of assay leaves a footprint on the plate that can be read as a blue spot. The number of spots on the plate were counted under a magnification of 3 to determine the number of ISC per $10^{6}$ mononuclear cells. For ISC at days 3 and $6,10^{6}$ cells per $\mathrm{ml}$ were cultured in round bottom $12 \times 75$ tubes (Fisher Scientific Co., Pittsburgh, PA) in complete medium. On the day of assay, the cells were washed twice in RPMI and set up in RPMI and $1 \%$ BSA as described above.

Lymphokine secretion. To assay production of IL 2 and IFN $\gamma 2$ $\times 10^{6} \mathrm{~T}$ cells per $\mathrm{ml}$ were placed in complete media, with and without 2 $\mu \mathrm{g} / \mathrm{ml}$ PHA. Cells were cultured in flasks and supernatants removed after various times in culture. Supernatants were filtered and stored at $-20^{\circ} \mathrm{C}$ until assayed for IL 2 or IFN $\gamma$.

Assay for IL 2 and IFNy. IL-2 activity was measured using CTLL cells, a murine IL 2-dependent T cell line (kind gift of Dr. Paul Allen, Washington University School of Medicine). $4 \times 10^{3}$ cells per well were cultured in 96-well flat-bottom microtiter plates in complete media for $36 \mathrm{~h}$ with and without $\mathrm{T}$ cell supernatants. Known amounts of recombinant IL 2 (kind gift of Dr. E. S. Medlock, Amgen Corp., Thousand Oaks, CA) were used as standards. In some experiments PC61 and 7D4, antibodies to IL 2 receptor, were added to varying dilutions of $T$ cell supernatants in the CTLL assay $(11,12)$. Incorporation of tritiated thymidine was measured over the last $18 \mathrm{~h}$. T cell supernatants were assayed for IFN $\gamma$ by ELISA, using a MAb to human IFN $\gamma$ (kind gift of Dr. R. Schreiber, Washington University School of Medicine) as described (13).

Statistical analyses were performed using $t$ test. Data are presented as mean \pm SEM unless otherwise stated.

\section{Results}

Normal INT MNC express more surface activation antigens than PBMC. Donor small and large INT MNC and PBMC were compared for expression of 4F2, 5E9, and anti-TAC. As seen in Fig. 1, all three activation antigens were expressed on normal INT MNC immediately after isolation, before in vitro tissue culture. MNC from normal donor large intestine expressed more surface activation antigens than did MNC from normal small intestine but this was not statistically significant. In contrast, PBMC expressed significantly fewer activation antigens, compared with INT MNC from either small or large intestine $(P<0.05)$. PBMC preparations were treated with collagenase to examine whether the isolation procedure itself could induce enhanced expression of activation markers and there was no enhancement of antigen expression on PBMC (data not shown).

To identify which cell population(s) were activated two approaches were taken: intestinal $B$ and $T$ cells were isolated and stained with MAb (three specimens); and dual fluorescent markers were also performed on unseparated MNC populations (four specimens). Similar results were obtained using both methods. As seen in Fig. 2, both B and T cells from normal intestine expressed more 4F2, 5E9, and anti-TAC than $B$ and $T$ cells from peripheral blood. There were some differences between the populations with B cells having more 5E9 positive cells $(P<0.05)$ and T cells having more cells positive for $4 F 2$ (not statistically significant). Similar expression of anti-TAC was seen on both cell populations. The large and small INT MNC shown in Figs. 1 and 2 were derived from multiple different donor bowel specimens. Although results consistently showed increased expression of activation antigens, there was significant variation between individuals as shown by the wide standard errors. Therefore, we compared activation antigens on MNC from small and large bowel of the same three donors. When INT MNC from large and small bowel of the same normal donors were studied, large INT MNC expressed significantly more $4 \mathrm{~F} 2$, anti-TAC and BA5 than small INT MNC (Table I), as Figs. 1 and 2 suggested. In

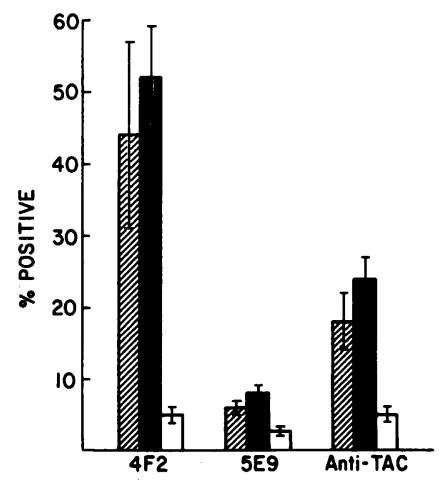

Figure 1. Mean expression of cell surface activation antigens on freshly isolated MNC from small intestine ( $\square)$, large intes tine ( () , and peripheral blood (ם). Mean \pm SEM of seven experiments showing the percentage of cells staining positive using MAb 4F2, 5E9 and anti-TAC. 


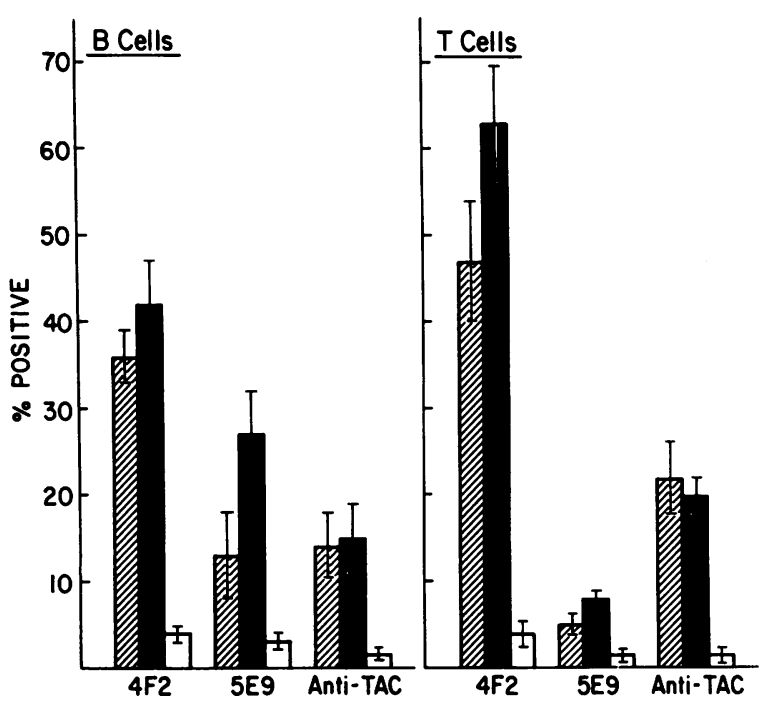

Figure 2. In vivo expression of cell surface activation antigens on $B$ and $T$ cells from small intestine ( $($ ) $)$ large intestine $(\square)$, and peripheral blood ( $\square)$. Mean \pm SEM of seven experiments.

each sample, large INT MNC expressed more activation antigens than small INT MNC except in one specimen in which 5E9 was expressed more on small INT MNC. The differences were significant for 4F2, anti-TAC, and BA5 by $t$ test and paired $t$ test (the latter data shown in Table I). There was no difference in the percentages of $B$ and $T$ cells and monocytes as assessed by MAb staining. Thus, INT MNC derived from normal intestinal lamina propria have increased expression of cell surface activation markers on both $T$ and $B$ cells and this expression appears to be higher in the large intestine than the small intestine.

We next investigated whether INT MNC were maximally activated or could be further induced to express more surface antigens with time in culture with PWM. Kinetic experiments with peripheral blood have shown that after activation, the 4F2-defined antigen appears at $24 \mathrm{~h}$ and the transferrin receptor and IL 2 receptor are maximal at $72 \mathrm{~h}(14)$ and then decline with time in culture $(15,16)$ over the next $72 \mathrm{~h}$. Fig. 3 shows the expression of $4 \mathrm{~F} 2$ and anti-TAC at time zero and after 3 and $5 \mathrm{~d}$ in vitro culture with PWM. Cell viability at days 3 and 5 was $>90 \%$. Both $4 \mathrm{~F} 2$ and anti-TAC expression on normal small INT MNC increased over $3 \mathrm{~d}$ in culture and then declined. As noted before, normal large INT MNC appeared more activated in vivo than small INT MNC. 4F2 expression increased over $3 \mathrm{~d}$ and anti-TAC expression was maximal in vivo and decreased with time in culture. In contrast, PBMC

Table I. INT MNC Activation Markers

\begin{tabular}{lccc}
\hline & $\begin{array}{c}\text { SI MNC } \\
(\% \text { positive })\end{array}$ & $\begin{array}{c}\text { LI MNC } \\
(\% \text { positive })\end{array}$ & $\begin{array}{c}\text { P value } \\
\text { (paired t test) }\end{array}$ \\
\hline 4F2 & $28.5 \pm 7.0$ & $38.6 \pm 2.5$ & $<0.025$ \\
Anti-TAC & $12.6 \pm 5.6$ & $23.7 \pm 6.0$ & $<0.05$ \\
5E9 & $5.9 \pm 2.0$ & $8.5 \pm 2.0$ & NS \\
BA5 & $10.3 \pm 0.6$ & $18.5 \pm 3.0$ & $<0.025$ \\
\hline
\end{tabular}

INT MNC from small (SI) and large (LI) intestine of the same three donors. Mean \pm SD.

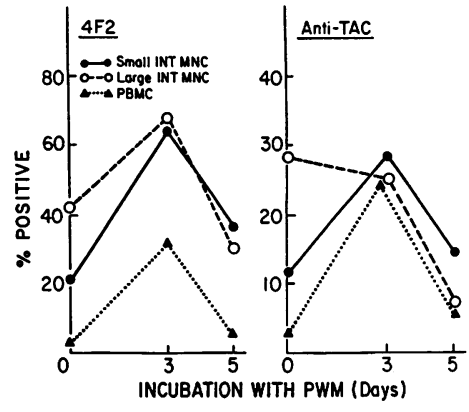

Figure 3. Expression of cell surface activation antigens on MNC from small intes-

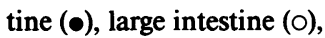
and peripheral blood ( $\Delta)$. Percentage of cells staining positive with $4 \mathrm{~F} 2$ (left) and anti TAC (right) were determined after isolation (time zero) and after 3 and $5 \mathrm{~d}$ in culture with PWM.

contained very few in vivo activated cells at time zero but 4F2 and anti-TAC was induced after in vitro stimulation with PWM.

Lymphokine secretion. To assess the activity of $\mathrm{T}$ lymphocytes, we studied the amount of IL 2 and gamma interferon secreted over a 48-h culture period by INT T cells. Unstimulated cultures produced no detectable IL 2. After PHA stimulation, maximum IL 2 secretion was seen at 4-8 h with a decrease over the ensuing 24-48 h (Fig. 4). No IL 2 secretion was detected before $4 \mathrm{~h}$. PHA alone did not stimulate CTLL cells to proliferate. Antibody to IL 2 receptor inhibited proliferation of $30 \mathrm{U}$ recombinant IL 2 . When IL 2 receptor antibody was added to varying dilutions of the 4-8-h $\mathrm{T}$ cell supernatants in the three experiments shown in Fig. 4, maximal $\left[{ }^{3} \mathrm{H}\right]$ thymidine incorporation decreased to background in two experiments and decreased by $75 \%$ in the third experiment. Thus some, but not all, of the $T$ cell growth activity secreted by stimulated INT T cells was due to IL 2 .

These same specimens were studied for their ability to secrete IFN $\gamma$. No IFN $\gamma$ was secreted by unstimulated T cells. Only one of three intestinal $\mathrm{T}$ cell supernatants had significant levels ( $>10 \mathrm{IU} / \mathrm{ml}$ ) of IFN $\gamma$ after PHA stimulation in vitro. In this sample there were $29 \mathrm{IU} / \mathrm{ml}$ at $24 \mathrm{~h}$ and $62 \mathrm{IU} / \mathrm{ml}$ at $36 \mathrm{~h}$. Thus IFN $\gamma$ is not secreted by unstimulated intestinal $\mathrm{T}$ cell cultures and is variably secreted by PHA stimulated INT T cell cultures.

Normal human INT MNC spontaneously secrete antibody in vitro without addition of antigen or mitogen. INT MNC derived from the large and small bowel of the same normal donors were cultured for $14 \mathrm{~d}$ and supernatants tested for secreted IgA, IgG, and IgM. As can be seen in Fig. 5, there was marked secretion of IgA, moderate secretion of $\mathrm{IgG}$ and less secretion of IgM after $14 \mathrm{~d}$ in culture. Normal large bowel INT MNC secreted more IgA than normal small bowel INT MNC $(P<0.001)$. There was no difference in IgG and IgM secretion

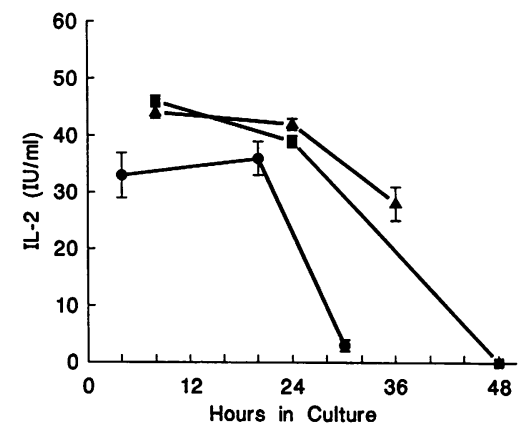

Figure 4. IL 2 secretion by INT T cells. $2 \times 10^{6}$ INT T cells/ml were cultured with $2 \mu \mathrm{g} / \mathrm{ml}$ PHA. Supernatants were removed at indicated times and tested for IL 2 production in CTLL assay. Mean \pm SD of triplicate wells. 


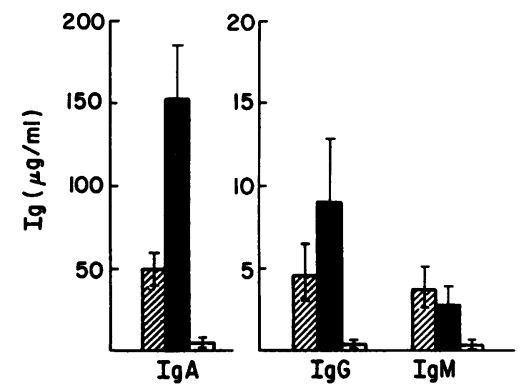

Figure 5. Secretion of IgA, IgG, and IgM by MNC from small intestine (四), large intestine $(\square)$, and peripheral blood (口) cultured for $14 \mathrm{~d}$ in media alone. Mean \pm SEM of 12 experiments.

between large and small bowel. In comparison, unstimulated PBMC secreted $<0.5 \mu \mathrm{g} / \mathrm{ml}$ over a 14-d culture period.

We then studied the kinetics of immunoglobulin secretion by INT MNC from six intestinal specimens. As shown in Fig. 6 on a logarithmic scale, there was significant early secretion of IgA during the initial 48-h culture, and this increased further over the ensuing $10 \mathrm{~d}$. IgA secretion increased significantly from days 4 to 8 , and from days 8 to 14 in culture $(P<0.03$ paired $t$ test). IgG secretion increased significantly until day 8 $(P<0.03)$ and then plateaued. IgM secretion did not significantly increase after day 4 in culture (day 4-14: $P=0.057$ ). Again IgA was the predominant isotype secreted, with less IgG and small amounts of IgM.

To further define this enhanced spontaneous antibody secretion, the number of ISC were estimated immediately after isolation by the spot ELISA technique. This assay reflects the number of B cells that have differentiated into antibody-secreting plasma cells. Even without in vitro culture (time zero) spontaneous ISC of all isotypes were present in normal INT MNC (Fig. 7). There were more IgA-secreting cells in the large intestine than the small intestine $(P<0.05)$, whereas there was no difference in IgG- or IgM-secreting cells. In contrast, PBMC contained very few B cells spontaneously secreting Ig at time zero (Fig. 7). To control for the effects of the isolation procedure on the secretion of Ig by MNC, PBMC were subjected to the same conditions as used for INT MNC isolation. This treatment did not induce ISC by PBMC (data not shown).

The kinetics of in vitro induction of ISC by intestinal MNC populations were determined over a 6-d culture period (Fig. 8). ISC were detected at time zero and after 3 and $6 \mathrm{~d}$ in culture. There were significantly more IgA ISC at time zero than ISC of IgG or IgM isotype $(P<0.02)$. After $3-6 \mathrm{~d}$ in vitro culture, more IgA-secreting B cells had differentiated into plasma cells $(P<0.05)$. In contrast, IgG and IgM ISC decreased with further time in culture (IgG ISC $P<0.05$; IgM ISC decrease not significant). Thus there were $B$ cells differentiating over 3 and $6 \mathrm{~d}$ into Ig-secreting plasma cells, predominantly of the IgA isotype. In marked contrast, PBMC had less than 100 ISC per

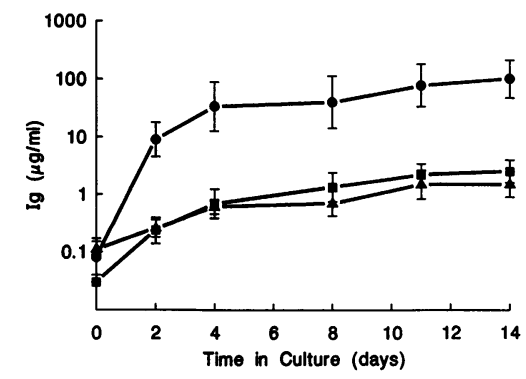

Figure 6. Kinetics of $\operatorname{IgA}(\bullet), \operatorname{IgG}(\boldsymbol{(})$, and $\operatorname{IgM}(\boldsymbol{\Lambda})$ secretion by INT MNC over $14 \mathrm{~d}$ in culture. Geometric mean $\times$ SEM of six normal intestinal specimens cultured in media alone.

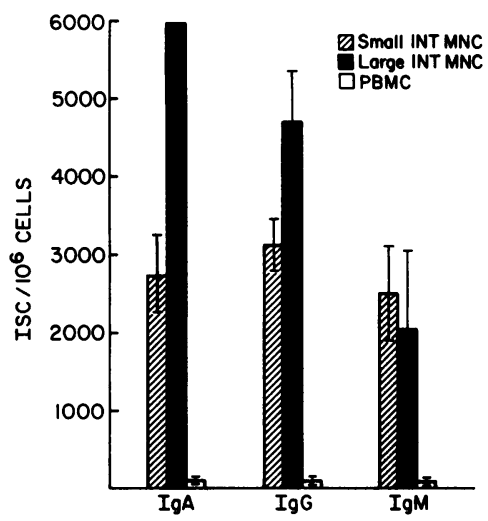

Figure 7. ISC per million freshly isolated MNC from small intestine ( $\square)$, large intestine ( $\square)$, and peripheral blood (口). Mean \pm SD of nine experiments.

million cells both at time zero and in unstimulated cultures. ISC were only noted in PBMC cultures which had been stimulated for $6 \mathrm{~d}$ with PWM (data not shown).

To look at the influence of other cell types on B cells, we measured IgA secreted in 14-d culture supernatant from unfractionated (UF) and monocyte-depleted INT MNC and found no difference in the amount of immunoglobulin secreted (Table II, section A). We then compared unfractionated INT MNC with B cell-enriched populations. As shown in Table II, section B, there was no difference in the number of IgA-secreting cells between unfractionated and B cell-enriched populations, when adjusted to show the number of ISC per $10^{6}$ $B$ cells. In addition, after $6 \mathrm{~d}$ in culture, the increase in IgA-secreting cells was similar in unfractionated and B cell-enriched populations. We also found no difference in ISC when mixed lymphocyte culture supernatants were used as a source of $T$ cell-derived factors to induce ISC by B cell enriched populations at day 3 and 6 (data not shown). We then compared the effect of T cells on ISC from isolated B cells (Table II, section B) and found no significant effect of T cells on time zero ISC. Unfortunately, there were insufficient cells to deplete both monocytes and $T$ cells in the same experiment. Taken together, these data show that some INT B cells are sufficiently activated to secrete $\mathrm{Ig}$ at time zero and after $6 \mathrm{~d}$ in culture. Monocytes and $\mathrm{T}$ cells are not required for time zero ISC.

Thus, INT B cells are activated in vivo and spontaneously secrete Ig in vitro. Some preactivated B cells from spleen and peripheral blood have been shown to express $\operatorname{CD5}(17,18)$. We therefore separated the B-cell enriched population (56\% positive for B cells) on the basis of Leu 1 by cell sorting to determine whether the CD5-positive or CD5-negative INT B cells were responsible for spontaneous $\mathrm{Ig}$ secretion at time zero. $55 \%$ of the starting B cell enriched population were CD5 positive, including cells that stained dull and bright by fluorescence. Populations of CD5 bright positive and CD5 negative $B$

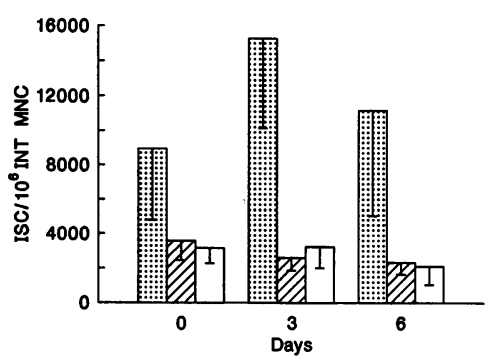

Figure 8. Kinetics of ISC as measured by spot ELISA. IgA ( $(0)$, IgG (D) and IgM (D) ISC were measured before culture (o) and after 3 and $6 \mathrm{~d}$ in culture. Mean \pm SEM of six experiments. 
Table II. Effect of Monocytes and T Cells on ISC and Ig Secretion

\begin{tabular}{lcc}
\hline \multicolumn{2}{c}{ INT MNC preparation } & \\
\hline & \multicolumn{2}{c}{ Secreted IgA (ng/ml) } \\
\cline { 2 - 3 } A UF & $5,248 \pm 1,201$ & \\
Monocyte depleted & $6,977 \pm 1,133$ & Day 6 \\
& IgA secreting cells $/ 10^{6}$ B cells \\
\cline { 2 - 3 } B & Day 0 & $13,560 \pm 3,310$ \\
UF & $5,630 \pm 550$ & $16,000 \pm 1,420$ \\
B cells & $6,800 \pm 260$ & \\
C & & \\
B cells & $28,650 \pm 3,620$ & \\
B cells + T cells & $31,400 \pm 1,158$ & \\
\hline
\end{tabular}

A. Amount of IgA secreted by $10^{5}$ UF INT MNC (6\% monocytes) and $10^{5}$ monocyte depleted INT MNC ( $1 \%$ monocytes) after $10 \mathrm{~d}$ in culture. Mean $\pm S D$.

B. Number of ISC in unfractionated INT MNC populations (UF) and $B$ cell-enriched populations (B) at time zero and after $6 \mathrm{~d}$ in culture. Mean \pm SD.

C. Number of ISC in B cell-enriched (B) populations with and without equal numbers of $T$ cells. Mean $\pm S D$.

cells were obtained by sorting as shown in Fig. 9 and ISC were determined in both populations. The purified B cells contained $<5 \% \mathrm{~T}$ cells by $\mathrm{E}$ rosette and leu 4 (Becton Dickinson $\&$ Co.) staining. The CD5 bright positive B cells contained four times the number of IgA-secreting plasma cells than the CD5negative B cells. Both CD5 bright positive and negative populations contained 600 IgM- and $<100$ IgG-secreting cells per $10^{6} \mathrm{~B}$ cells. The CD5 dull positive population was not studied. Therefore, the total contribution from CD5-positive and negative populations to Ig-secreting cells cannot be calculated. However, in normal intestine CD5 (Leu 1) may be a marker for activated B cells, and these preactivated CD5 positive B cells predominantly secrete IgA.

\section{Discussion}

These studies describe the state of activation of intestinal B cells and show by three different criteria that these cells are activated: (a) there is an increase in expression of cell surface activation antigens, $(b)$ the presence of spontaneous ISC and (c) high spontaneous immunoglobulin secretion by unstimu- lated cells. The large intestine is more activated than the small intestine. The intestinal lamina propria mononuclear cells may be activated because of continued antigenic stimulation in response to the high levels of bacterial colonization especially in the large intestine. These studies investigate the mechanism of this $B$ cell activation by studying activation of $T$ cells and have shown that the $T$ cells themselves are activated. They also show for the first time that CD5-positive cells can be $\operatorname{IgA}$ positive and not just IgM-producing cells.

The level of continued antigenic stimulation will determine the percentage of the $B$ cell repertoire that is preactivated and no longer in the resting state. Intestinal MNC may have ongoing antigenic stimulation via the intestinal wall. There is an increase in the expression of surface activation antigens on both $B$ and $T$ cells from normal intestinal lamina propria. These same activation antigens have been shown to be induced on peripheral blood, splenic and tonsillar cells but only after stimulation in cell culture (14). $4 \mathrm{~F} 2$ is a $120-\mathrm{kD}$ surface protein that is maximally induced $24 \mathrm{~h}$ after activation (5). 5E9 is an antibody that recognizes the transferrin receptor, which is maximally expressed $72 \mathrm{~h}$ after stimulation of resting $B$ and $T$ cells (6). Anti-TAC is an antibody that recognizes the $55-\mathrm{kD}$ protein of the IL 2 receptor and is present on activated $B$ and T cells (19). In peripheral blood $<2 \%$ of mononuclear cells express these activation antigens in vivo. Expression can be induced in vitro and then declines with further time in culture (14-16). In marked contrast, a much higher percentage of INT $\mathrm{MNC}$ was found to be activated in vivo.

INT MNC secrete large amounts of $\mathrm{Ig}$, especially IgA, without stimulation by antigen or mitogen in culture. Large bowel INT MNC secrete more antibody than small bowel INT MNC. After isolation, before time in culture, there are markedly increased numbers of plasma cells in INT MNC compared with peripheral blood. While monocytes and $\mathrm{T}$ cells are required for $T$ cell-dependent $B$ cell activation, we did not find them necessary for Ig secretion by in vivo preactivated B cells. This may be due to prior in vivo $T$ cell and monocyte activation. The requirements for B cell activation, proliferation, and differentiation have been well characterized in man, using peripheral blood and tonsillar MNC (20). Studies using PBMC from normal individuals immunized to protein antigens have revealed that the in vitro responses of peripheral blood B cells depend on the length of time from recall or booster immunization (21, 22). 5-7 d after booster immunization, peripheral blood contains B cells capable of spontaneous antibody secretion without addition of $T$ cell factors or mitogen (23). These lymphoblastoid cells are $B$ cells that have terminally differen-

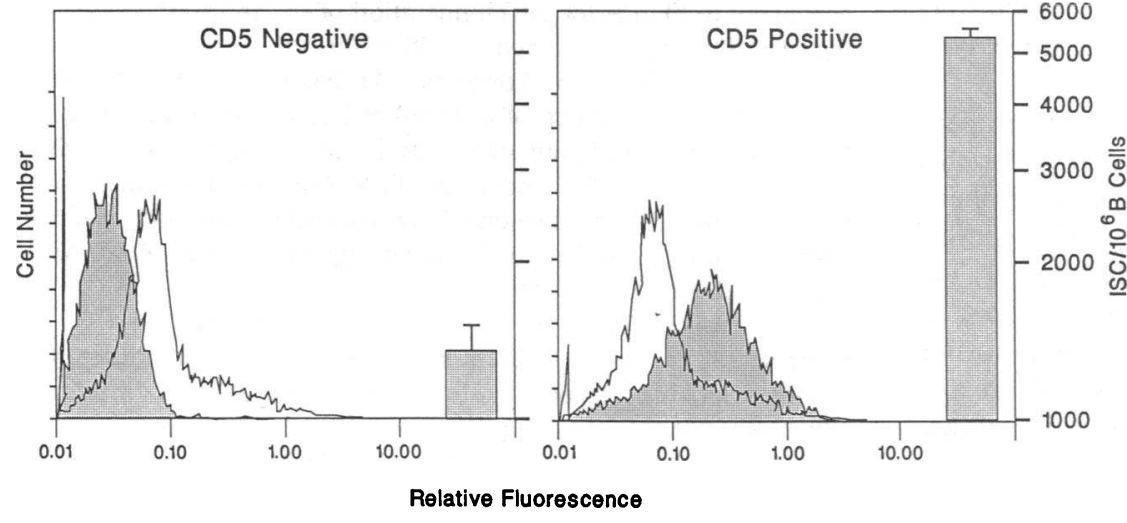

Figure 9. Plasma ISC from freshly isolated INT MNC are CD5 positive. INT MNC were $T$ cell depleted and CD5 bright positive (right) and negative (left) B cells were sorted by FACS. Mean \pm SD of number of IgA secreting cells per million B cells shown for both populations in shaded bar to right of each panel. 
tiated into antibody-secreting plasma cells. Two weeks after immunization another population of B cells appear that can terminally differentiate in vitro in response to $T$ cell factors. These in vivo preactivated $B$ cells can be driven to terminal differentiation with the addition of factors alone, and do not require further stimulation with antigen or mitogen. Further characterization revealed that B cell growth factor, B cell differentiation factor, and IL 2 were required for optimal differentiation of these in vivo preactivated B cells but other factors such as alpha IFN can modulate Ig production $(22,24)$. These cells are present for only a finite period of time and 6 mo after immunization, specific B cell responses can only be obtained by mitogen stimulation in the presence of $\mathrm{T}$ cells and monocytes (22).

Intestinal B lymphocytes are already preactivated and secrete large amounts of $\operatorname{IgA}$ and $\operatorname{IgG}$ in vitro without further addition of antigen or mitogen. INT MNC contain both activated B cells and plasma cells. Plasma cells are estimated by ISC at time zero and preactivated B cells are cells that require time in culture to differentiate into Ig-secreting plasma cells. In contrast to the secreted antibody that was predominantly $\operatorname{IgA}$, all three isotypes (IgG, IgA, and IgM) were represented at time zero. These in vivo stimulated plasma cells expressed CD5 on their cell surface. Although a common $T$ cell antigen, this activation marker has also been found on B cells from peripheral blood and spleen that secrete antibody to rheumatoid factor and ssDNA $(17,18)$. These autoantibodies from spleen and peripheral blood were of the IgM isotype. INT CD5-positive B cells were predominantly IgA in keeping with the predominant mucosal isotype found. Thus INT B cells are a highly activated but heterogeneous population containing lymphoblastoid cells, and preactivated B cells capable of differentiation with time in culture.

This was in marked contrast to peripheral blood. Normal PBMC have very few ISC at time zero but they can be induced in vitro after stimulation with mitogen or antigen. Increased time zero ISC have been found in PBMC but only in disease states from individuals with SLE or AIDS $(1,25)$. However, ISC at time zero are found in normal intestinal lamina propria lymphocytes, more from the large than the small intestine.

Normal INT MNC also contain activated T cells. There is increased expression of activation antigens in agreement with nonhuman primate data (26). Supernatants from PHA stimulated INT T cells have T cell growth factor activity, which is maximal after 4-8 h of culture. This is in contrast to tonsillar cells that have detectable IL 2 activity at $8 \mathrm{~h}$ but maximal accumulation at $24 \mathrm{~h}$ (27). The CTLL line responds to human IL 2. Our inhibition studies with antibody to IL 2 receptor suggest that IL 2 can be secreted by INT T cells but further studies however would be required to categorize other lymphokines secreted by INT MNC.

The normal intestinal lamina propria lymphoid compartment has a distinct state of activation compared with other lymphoid compartments such as spleen, tonsil, and peripheral blood. Only the bone marrow appears to show similar spontaneous activation as shown by 14-d in vitro culture (3). The high spontaneous antibody secretion by INT MNC is not reflected in the peripheral blood or spleen of the same normal individuals. Not only are B cells activated, but $\mathrm{T}$ cells are also activated. These B cells are capable of marked secretion of IgA, and some secretion of IgG and IgM. This heightened state of activation by intestinal lamina propria lymphoid compartment may be important for its distinct role in mucosal defense.

\section{Acknowledgments}

The authors wish to thank Dr. Robert Schreiber for assistance with IFN assays, Dr. Gwen Mazoujin for Factor VIII and keratin antibodies, Dr. Eric Brown for critical review of the manuscript.

This work was supported in part by U. S. Public Health Service grants DK-33487; DK-21474; the National Foundation for Ileitis and Colitis; and Washington University Monsanto Biochemical Agreement.

\section{References}

1. MacDermott, R. P., G. S. Nash, M. J. Bertovich, M. V. Seiden, M. J. Bragdon, and M. G. Beale. 1981. Alterations of IgM, IgG, and IgA synthesis and secretion by peripheral blood and intestinal mononuclear cells from patients with ulcerative colitis and Crohn's disease. Gastroenterology. 81:844-852.

2. Bøyum, A. 1968. Isolation of mononuclear cells and granulocytes from human blood. Scand. J. Clin. Lab. Invest. 23:31-42.

3. MacDermott, R. P., G. S. Nash, M. J. Bertovich, R. F. Mohrman, I. J. Kodner, D. L. Delacroix, and J.-P. Vaerman. 1986. Altered patterns of secretion of monomeric IgA and IgA subclass 1 by intestinal mononuclear cells in Inflammatory Bowel Disease. Gastroenterology. 91:379-385.

4. Falkoff, R. M., M. Peters, and A. S. Fauci. 1982. T cell enrichment and depletion of human peripheral blood mononuclear cell preparations. Unexpected findings in the study of the functional activities of the separated populations. J. Immunol. Methods. 50:33-49.

5. Haynes, B. F., M. E. Hemler, D. L. Mann, G. S. Eisenbarth, J. Shelhamer, H. S. Mostowski, C. A. Thoms, J. L. Strominger, and A. S. Fauci. 1981. Characterization of a monoclonal antibody (4F2) that binds to human monocytes and to a subset of activated lymphocytes. $J$. Immunol. 126:1409-1414.

6. Haynes, B. F., M. Hemler, T. Cotner, D. L. Mann, G. S. Eisenbarth, J. L. Strominger, and A. S. Fauci. 1981. Characterization of a monoclonal antibody (5E9) that defines a human cell surface antigen or cell activation. J. Immunol. 127:347-351.

7. Uchiyama, T., S. Broder, and T. A. Waldmann. 1981. A monoclonal antibody (Anti-Tac) reactive with activated and functionally mature human T cells. J. Immunol. 126:1393-1397.

8. Ambrus, J. L., C. H. Jurgensen, E. J. Brown, P. McFarland, and A. S. Fauci. 1988. Identification of a receptor for high molecular weight human B cell growth factor. J. Immunol. 141:861-869.

9. Lane, H. C., D. J. Volkman, G. Whalen, and A. S. Fauci. 1981. In vitro antigen induced, antigen-specific antibody production in man. J. Exp. Med. 154:1043-1057.

10. Sedgwick, J. D., and P. G. Holt. 1983. A solid-Phase immunoenzymatic technique for the enumeration of specific antibody-secreting cells. J. Immunol. Methods. 57:301-309.

11. Ceredig, R., J. W. Lowenthal, M. Nabholz, and H. R. MacDonald. 1985. Expression of interleukin-2 receptors as a differentiation marker on intrathymic stem cells. Nature (Lond.). 314:98-100.

12. Malek, T. R., R. J. Robb, and E. M. Shevach. 1983. Identification and initial characterization of a rat monoclonal antibody reactive with the murine interleukin 2 receptor-ligand complex. Proc. Natl. Acad. Sci. USA. 80:5694-5698.

13. Leinikki, P. O., J. Calderon, M. H. Luquette, and R. D. Schreiber. 1987. Reduced receptor binding by a human interferon- $\gamma$ fragment lacking 11 carboxyl-terminal amino acids. J. Immunol. 139:3360-3366.

14. Kehrl, J. H., A. Muraguchi, and A. S. Fauci. 1984. Differential 
expression of cell activation markers after stimulation of resting human B lymphocytes. J. Immunol. 132:2857-2861.

15. Neckers, L. M., G. Yenokida, and S. P. James. 1984. The role of the transferrin receptor in human B lymphocyte activation. J. Im munol. 133:2437-2441.

16. Cantrell, D. A., and K. A. Smith. 1983. Transient expression of interleukin 2 receptors. Consequences for T cell growth. J. Exp. Med. 158:1895-1911.

17. Casali, P., S. E. Burastero, M. Nakamura, G. Inghirami, and A. L. Notkins. 1987. Human lymphocytes making rheumatoid factor and antibody to ssDNA belong to Leu- ${ }^{+}$B-cell subset. Science (Wash. DC). 236:77-83.

18. Taniguchi, O., H. Miyajima, T. Hirano, M. Noguchi, A. Ueda, H. Hashimoto, S.-I. Hirose, and K. Okumura. 1987. The Leu-1 B-cell subpopulation in patients with rheumatoid arthritis. J. Clin. Immunol. 7:441-448.

19. Muraguchi, A., J. H. Kehrl, D. I. Longo, D. J. Volkman, K. A. Smith, and A. S. Fauci. 1985. Interleukin 2 receptors on human B cells. Implications for the role of Interleukin 2 in human B cell function. J. Exp. Med. 161:181-197.

20. Kehrl, J. H., A. Muraguchi, J. L. Butler, R. J. M. Falkoff, and A. S. Fauci. 1984. Human B cell activation, proliferation and differentiation. Immunol. Rev. 78:75-96.

21. Peters, M., and A. S. Fauci. 1983. Selective activation of anti- gen-specific human B cells in recently immunized individuals by nonspecific factors in the absence of antigen. J. Immunol. 130:678-680.

22. Peters, M., J. L. Butler, J. B. Margolick, T. L. Gerrard, C. A. Dinarello, and A. S. Fauci. 1985. Synergy of helper factors in the differentiation of in vivo-preactivated antigen-specific human B cells. Cell. Immunol. 91:33-42.

23. Stevens, R. H., E. Macy, C. Morrow, and A. Saxon. 1979. Characterization of a circulating subpopulation of spontaneous antitetanus toxoid antibody producing B cells following in vivo booster immunization. J. Immunol. 122:2498-2504.

24. Peters, M., J. L. Ambrus, A. Zheleznyak, D. M. Walling, and J. H. Hoofnagle. 1986. Effect of alpha interferon on immunoglobulin synthesis by human B cells. J. Immunol. 137:3153-3157.

25. Lane, H. C., H. Masur, L. C. Edgar, G. Whalen, A. Rook, and A. Fauci. 1983. Abnormalities of B cell activation and immunoregulation in patients with the acquired immunodeficiency syndrome. $N$. Engl. J. Med. 309:453-458.

26. Zeitz, M., W. C. Greene, N. J. Peffer, and S. P. James. 1988. Lymphocytes isolated from the intestinal lamina propria of normal nonhuman primates have increased expression of genes associated with T-cell activation. Gastroenterology. 94:647-655.

27. Efrat, S., S. Pilo, and R. Kaempfer. 1982. Kinetics of induction and molecular size of mRNAs encoding human interleukin- 2 and $\gamma$-interferon. Nature (Lond.). 297:236-238. 